\title{
Patient-oriented education and medication management intervention for people with decompensated cirrhosis: study protocol for a randomized controlled trial
}

Kelly L. Hayward ${ }^{1,2}$, Jennifer H. Martin³ ${ }^{3}$ W. Neil Cottrell ${ }^{4}$, Antara Karmakar ${ }^{1}$, Leigh U. Horsfall ${ }^{5,6}$, Preya J. Patel ${ }^{5,6}$, David D. Smith ${ }^{7}$, Katharine M. Irvine ${ }^{5}$, Elizabeth E. Powell ${ }^{5,6+}$ and Patricia C. Valery ${ }^{{ }^{*}+}$

\begin{abstract}
Background: People with decompensated cirrhosis require complex medical care and are often prescribed an intricate and frequently changing medication and lifestyle regimen. However, many patients mismanage their medications or have poor comprehension of their disease and self-management tasks. This can lead to harm, hospitalization, and death.
\end{abstract}

Methods/design: A patient-oriented education and medication management intervention has been developed for implementation at a tertiary hospital hepatology outpatient center in Queensland, Australia. Consenting patients with decompensated cirrhosis will be randomly allocated to education intervention or usual care treatment arms when they attend routine follow-up appointments. In the usual care arm, participants will be reviewed by their hepatologist according to the current model of care in the hepatology clinic. In the intervention arm, participants will be reviewed by a clinical pharmacist to receive the education and medication management intervention at baseline in addition to review by their hepatologist. Intervention participants will also receive three further educational contacts from the clinical pharmacist within the following 6-month period, in addition to routine hepatologist review that is scheduled within this time frame. All participants will be surveyed at baseline and follow-up (approximately 6 months post-enrollment). Validated questionnaire tools will be used to determine participant adherence, medication beliefs, illness perceptions, and quality of life. Patients' knowledge of dietary and lifestyle modifications, their current medications, and other clinical data will be obtained from the survey, patient interview, and medical records. Patient outcome data will be collected at 52 weeks.

Discussion: The intervention described within this protocol is ready to adapt and implement in hepatology ambulatory care centers globally. Investigation of potentially modifiable variables that may impact medication management, in addition to the effect of a clinical pharmacist-driven education and medication management intervention on modifying these variables, will provide valuable information for future management of these patients.

Trial registration: Australian and New Zealand Clinical Trial Registry identifier: ACTRN12616000780459. Registered on 15 June 2016.

Keywords: Liver cirrhosis, Polypharmacy, Clinical pharmacist, Intervention study, Patient education, Medication reconciliation, Medication errors, Patient adherence, Medication adherence, Quality of life

\footnotetext{
* Correspondence: patricia.valery@qimrberghofer.edu.au

${ }^{\dagger}$ Equal contributors

${ }^{8}$ Cancer and Chronic Disease Research Group, Level 4, Central, QIMR

Berghofer Medical Research Institute, 300 Herston Rd, Brisbane, QLD 4006,

Australia

Full list of author information is available at the end of the article
} 


\section{Background}

The morbidity and health care costs associated with decompensated cirrhosis are substantial because patients require complex medical care to manage debilitating complications of their disease $[1,2]$. People with decompensated cirrhosis are often prescribed multiple medications for therapeutic or prophylactic use to reduce the negative health effects of cirrhosis. However, many patients lack the knowledge and skills required to contribute effectively to disease management $[3,4]$.

On average, this patient group has approximately three hospital admissions each year $[1,5]$, reflecting the high burden of illness and use of health care resources. The number of medications prescribed on hospital discharge has been found to predict rehospitalization rate and time to first hospital readmission, independently of serum biomarkers that also predict poor outcomes [2]. With recurrent admissions, more medicines are prescribed and/or the medication regimen is altered. This increases opportunities for miscommunication and nonadherence, thereby increasing the risk of medication errors and rehospitalization.

Poor knowledge of cirrhosis, medications, self-monitoring, and important dietary and lifestyle modifications has been described in ambulatory Australian patients with cirrhosis [4]. Low medication adherence was identified in over onefourth of patients $(27.5 \%)$, and discrepancies among conventional medications were also seen in $54.0 \%$ of patients with cirrhosis attending general hepatology clinics [6]. Communication barriers between patients and clinicians are a recognized impediment to patient care that may result in clinically significant discrepancies and other medication-related problems (MRPs) [7]. MRPs are events or circumstances involving medications that actually or potentially interfere with an optimum outcome of care [8]. They are complex and multifaceted issues that may be the result of numerous interacting factors. Examples of MRPs that may occur in patients with decompensated cirrhosis are presented in Table 1. It has

Table 1 Potential medication-related problems in patients with decompensated cirrhosis

\begin{tabular}{|c|c|c|}
\hline Classification and definition & Subtype & Example \\
\hline \multicolumn{3}{|l|}{ Adverse drug reaction } \\
\hline \multirow{3}{*}{$\begin{array}{l}\text { A medical problem resulting from an adverse effect of a drug. } \\
\text { These include sensitivities, intolerances, and immune-mediated } \\
\text { hypersensitivity reactions. }\end{array}$} & Minor & $\begin{array}{l}\text { Minor dizziness related to propranolol; manage with } \\
\text { lifestyle counseling. }\end{array}$ \\
\hline & Moderate & $\begin{array}{l}\text { Gynecomastia related to spironolactone; may require } \\
\text { dose adjustment or cessation. }\end{array}$ \\
\hline & Severe & $\begin{array}{l}\text { Stevens-Johnson syndrome precipitated by } \\
\text { sulfamethoxazole-trimethoprim (Bactrim DS }{ }^{\oplus} \text {. }\end{array}$ \\
\hline \multicolumn{3}{|l|}{ Drug interactions } \\
\hline \multirow[t]{2}{*}{$\begin{array}{l}\text { An actual or potential medical problem that is related to a } \\
\text { drug-drug or drug-patient interaction. }\end{array}$} & Drug-drug & $\begin{array}{l}\text { Harvoni }{ }^{\oplus} \text { (Gilead Sciences, Foster City, CA, USA) and } \\
\text { amiodarone. }\end{array}$ \\
\hline & Drug-patient & Hepatorenal syndrome precipitated by NSAID use. \\
\hline \multicolumn{3}{|l|}{ Drug use without indication } \\
\hline The patient is taking a medication for no medically valid reason. & & $\begin{array}{l}\text { Proton pump inhibitor use in a patient without } \\
\text { gastroesophageal reflux disease, peptic ulcer, or } \\
\text { variceal bleeding. }\end{array}$ \\
\hline \multicolumn{3}{|l|}{ Incorrect dosage } \\
\hline \multirow[t]{2}{*}{$\begin{array}{l}\text { A medical condition that is being treated with drug therapy; } \\
\text { however, the dose may be too low or too high. }\end{array}$} & Subtherapeutic & $\begin{array}{l}\text { 10-ml daily dose of lactulose, achieving one bowel } \\
\text { motion every second day. }\end{array}$ \\
\hline & Supratherapeutic & $\begin{array}{l}\text { Spironolactone } 400 \mathrm{mg} \text { daily in a patient with } \\
\text { minimal abdominal ascites. }\end{array}$ \\
\hline \multicolumn{3}{|l|}{ Nonadherence } \\
\hline \multirow{2}{*}{$\begin{array}{l}\text { The patient is prescribed a drug for a medical condition but is } \\
\text { not taking it for psychological, sociological, or economic reasons. }\end{array}$} & Unintentional & Forgetting to take propranolol at nighttime. \\
\hline & Intentional & $\begin{array}{l}\text { Not taking lactulose because of side effects } \\
\text { (flatulence, bloating) or cost following removal } \\
\text { from the Pharmaceutical Benefits Scheme. }\end{array}$ \\
\hline \multicolumn{3}{|l|}{ Untreated indications } \\
\hline \multirow{2}{*}{$\begin{array}{l}\text { A medical condition that requires drug therapy but is not being } \\
\text { treated with medication. This may be related to intentional or } \\
\text { unintentional nonadherence by the patient, or to intentional or } \\
\text { unintentional underprescribing by a medical practitioner. }\end{array}$} & Nonadherence & As above. \\
\hline & Underprescribing & $\begin{array}{l}\text { Low cholecalciferol and tocopherol identified } \\
\text { by pathology, not supplemented. } \\
\text { Unintentional - oversight. } \\
\text { Intentional - patient unable to afford currently; } \\
\text { to be reconsidered at next visit. }\end{array}$ \\
\hline
\end{tabular}


been estimated that up to $22 \%$ of 30 -day readmissions among patients with decompensated cirrhosis may be preventable with improved medication management or more frequent monitoring [2].

In existing models of collaborative outpatient practice, integrated pharmacist education and medication management interventions have been shown to improve patient knowledge, adherence, and outcomes in chronic diseases that have complex medication management issues similar to those of cirrhosis [9-13]. Identification of medication discrepancies and MRPs using a clinical pharmacist's reconciled medication record reduces harm and hospitalization and may allow for simplification of the prescribed regimen [14, 15]. The association between patient knowledge, polypharmacy, poor adherence, and medication discrepancies has not been investigated in patients with decompensated cirrhosis to date. It is hypothesized that addressing this complicated relationship by implementing a targeted patient education and medication management intervention in a multidisciplinary model of hepatology ambulatory care may improve medication management, reduce MRPs, and improve medication-related outcomes for people with decompensated disease.

\section{Methods/design}

\section{Study aims and hypotheses}

The primary aim of the study is to determine whether the intervention (medication review, reconciliation, and additional education by a clinical pharmacist) reduces the frequency (counts) and severity (measure of clinical significance) of discrepancies between patient-reported and medical record-documented "current" medications. The secondary aims are to describe the impact of the intervention on medication adherence, quality of life (QoL), medication and illness beliefs, patient knowledge of disease and self-management tasks related to decompensation history and prescribed therapy, and the frequency of hospitalization for management of cirrhosisrelated complications, non-cirrhosis-related complications, and survival. The sustainability of the intervention in the current health care system in relation to costs and cost-effectiveness will also be evaluated.

\section{Hypotheses}

Compared with patients receiving usual care, patients who receive the tailored (additional) education and medication management intervention are hypothesized to benefit in the following ways:

(H1) Fewer medication discrepancies

(H2) Improved medication adherence

(H3) Improved QoL

(H4) Improved illness perceptions

(H5) Improved medication beliefs
(H6) Improved knowledge of disease self-management tasks related to prescribed medications

(H7) Fewer hospital admissions

(H8) Longer survival time

\section{Design and setting}

The study has a randomized, controlled, parallel-group design and will be conducted at a single site, the Hepatology Outpatient Centre at the Princess Alexandra Hospital (PAH), located in Brisbane, Australia. The Gastroenterology and Hepatology Department at the $\mathrm{PAH}$ is one of the largest hepatology centers in Australia and the only liver transplant center in Queensland. The hospital's local catchment area is $3,856 \mathrm{~km}^{2}$. Additionally, many patients travel from regional and remote areas, including interstate to access specialist services. Consequently, the Hepatology Outpatient Centre is responsible for the ambulatory care of a significant proportion of southeastern Queensland patients with chronic liver disease (CLD).

\section{Participants \\ Eligibility criteria}

Eligible participants are (1) adults aged $\geq 18$ years; (2) diagnosed with hepatic cirrhosis by a hepatologist; and (3) current or recent (within the preceding 2 years) chronic liver failure-related complication(s), including ascites, variceal bleeding, spontaneous bacterial peritonitis, sepsis, encephalopathy, or liver-related renal dysfunction. Liver biopsy, ultrasound, and FibroScan ${ }^{\circ}$ technology (Echosens, Paris, France) will be used to confirm the diagnosis of cirrhosis if available [16, 17]. Patient medical records and correspondence letters will be used to identify history of chronic liver failurerelated complications.

\section{Exclusion criteria}

Individuals will be excluded for (1) inability to provide informed consent and/or (2) receiving intensive management by other health care teams (i.e., liver transplant team, palliative care).

\section{Participant recruitment}

The Hepatology Outpatient Centre's appointment database will be prescreened for eligible participants who are scheduled for routine review by a hepatologist. Persons identified as eligible will be contacted via telephone in the week preceding their appointment to discuss the study and offer participation. Participants will be given instructions to arrive 15 minutes early for their appointment, bring all medications to the clinic, and ask their carer or responsible family member to accompany them if appropriate. Consented participants will be randomly 
allocated to one of the two study arms: (1) intervention or (2) usual care.

\section{Randomization and allocation}

An independent person at the PAH will prepare individual envelopes labeled with the randomization number and containing the corresponding study arm. Allocation concealment will be achieved by use of sequentially numbered, sealed, opaque envelopes that will be stored and opened in order by a third party located within PAH.

\section{Study navigation}

Figure 1 describes the planned study flow, including data collection time points. A Standard Protocol Items: Recommendations for Interventional Trials (SPIRIT) checklist of standard protocol items [18] addressed within the study protocol is provided in Additional file 1.

All participants will complete a baseline survey prior to their scheduled outpatient clinic review. Validated questionnaire tools will be used to determine baseline medication adherence, medication beliefs, illness perceptions, and QoL, in addition to patient knowledge of important dietary and lifestyle modifications. The survey will be completed with a study coordinator over the telephone or in person in a private clinic room, or it will be self-completed by the participant in the clinic waiting room. Patient carers or family members will be involved if necessary.

Usual care participants will subsequently receive routine review and education by their hepatologist according to the current model of care in the hepatology clinic.

\begin{tabular}{|c|c|c|c|c|c|c|}
\hline \multirow[b]{3}{*}{ Time Point } & \multicolumn{6}{|c|}{ STUDY PERIOD } \\
\hline & \multicolumn{2}{|c|}{ Baseline } & \multicolumn{3}{|c|}{ Post Allocation } & \multirow{2}{*}{$\frac{\text { Close Ou }}{t_{4}}$} \\
\hline & $-t_{1}$ & $t_{0}$ & $t_{1}$ & $t_{2}$ & $t_{3}$ & \\
\hline \multicolumn{7}{|l|}{ ENROLMENT } \\
\hline \multirow{3}{*}{$\begin{array}{r}\text { Eligibility screen } \\
\text { Informed consent } \\
\text { Randomisation/Allocation }\end{array}$} & $\mathrm{X}$ & & & & & \\
\hline & $\mathrm{X}$ & & & & & \\
\hline & & $\mathrm{X}$ & & & & \\
\hline \multirow{5}{*}{$\begin{array}{l}\text { INTERVENTIONS } \\
\text { Intervention Participants } \\
\text { Medication reconciliation } \\
\text { Education provision } \\
\text { MRP identification } \\
\text { Usual Care Participants }\end{array}$} & & & & & & \\
\hline & & (:) & (:)/留 & (:)/筤 & (:) & \\
\hline & & (:) & ()/留 & (:)/留 & (:) & \\
\hline & & (:) & ()/繁 & (:)/䈪 & (:) & \\
\hline & & - & - & - & - & \\
\hline \multicolumn{7}{|l|}{$\begin{array}{l}\text { ASSESSMENTS } \\
\text { Survey endpoints: }\end{array}$} \\
\hline Adherence & $\mathrm{X}$ & & & & $\mathrm{X}$ & \\
\hline \multirow{2}{*}{$\begin{array}{l}\text { Medication Beliefs } \\
\text { Illness Perceptions }\end{array}$} & $X$ & & & & $\mathrm{X}$ & \\
\hline & $\mathrm{X}$ & & & & $\mathrm{X}$ & \\
\hline Quality of Life & $\mathrm{X}$ & & & & $\mathrm{X}$ & \\
\hline \multirow{2}{*}{$\begin{array}{r}\text { Knowledge/Health Literacy } \\
\text { Medication Discrepancies }\end{array}$} & $\mathrm{X}$ & & & & $\mathrm{X}$ & \\
\hline & $\mathrm{X}$ & $\mathrm{X}^{*}$ & & & $\mathrm{X}$ & \\
\hline \multirow{2}{*}{$\begin{array}{l}\text { Medication-Related Problems } \\
\text { Outcome data collation: }\end{array}$} & & $\mathrm{X}^{*}$ & $\mathrm{X}^{*}$ & $X^{*}$ & $\mathrm{X}^{*}$ & \\
\hline & & & & & & \\
\hline Hospitalisation & & & & & $\mathrm{X}$ & $\mathrm{X}$ \\
\hline \multirow{2}{*}{$\begin{array}{r}\text { Survival } \\
\text { Cost }\end{array}$} & & & & & $\mathrm{X}$ & $\mathrm{X}$ \\
\hline & & & & & & $\mathrm{X}$ \\
\hline
\end{tabular}

Key:

$-t_{1} \quad$ Less than 7 days before scheduled hepatology review

to Baseline review

$t_{1} \quad$ Follow-up contact (approximately 1 month)

$t_{2} \quad$ Follow-up contact (approximately 3 months)

$t_{3} \quad$ Follow-up contact (approximately 6 months, according to hepatology scheduling)

$t_{4} \quad$ Study close-out at week 52

$\mathrm{X} \quad$ Study action or measure

* Intervention participants only

(:) Face-to-face encounter between pharmacist and patient

Telephone encounter between pharmacist and patient

Fig. 1 Standard Protocol Items: Recommendations for Interventional Trials (SPIRIT) flow diagram of participant recruitment and study navigation. MRP Medication-related problem 
Intervention participants will be reviewed by a clinical pharmacist to receive the education and medication management intervention (described below) in addition to routine review and education by their hepatologist. Intervention participants will also receive three further educational contacts from the clinical pharmacist in addition to scheduled routine hepatologist review within the following 6-month period.

All participants will be resurveyed at follow-up $\left(t_{3}\right.$, approximately 6 months post-enrollment) to explore changes in their medication adherence, medication beliefs, illness perceptions, QoL, and knowledge. Patient outcome data will be collected at 52 weeks (see "Study endpoints and data collection" section below).

\section{Intervention development}

A multifaceted, clinical pharmacist-driven education and medication management intervention targeted to patients with decompensated cirrhosis was developed by a panel of clinicians (including a hepatologist, general physician/ pharmacologist, clinical nurse, and clinical pharmacist). Education and monitoring points (Table 2) were agreed upon on the basis of clinical hepatology guidelines to augment and support information provided by hepatologists and other clinicians involved in patient care.

\section{Intervention description}

During the initial encounter with each intervention patient (and carer/family member if present), the clinical pharmacist will obtain a complete, reconciled list of

Table 2 Medication-related education provided to intervention participants receiving pharmacotherapy for chronic liver failure-related complications

\begin{tabular}{lll}
\hline Clinical decompensation history & Advice & Monitoring \\
\hline Ascites & & \\
& Adherence with diuretics & Weigh self weekly if dose of diuretics is stable and ascites \\
& Do not add salt to food or when cooking & is well-controlled \\
Restaurant meals, take-out foods, and tinned foods & Weigh self daily if diuretic dose changes or poorly controlled \\
generally contain high levels of sodium & ascites; notify clinic if postural symptoms/dizziness develop \\
& Sea salt, Himalayan rock salt, and table salt have & Document weight - do not just "remember" \\
& similar sodium content & Do not restrict fluid intake unless advised to by a doctor \\
& Caution with salt substitutes_may contain high & Development of fevers and/or chills accompanied by \\
& levels of potassium & abdominal pain and/or discomfort-present to the emergency \\
& Avoid nonsteroidal anti-inflammatory analgesics & department for assessment \\
&
\end{tabular}

Encephalopathy

Hepatocellular carcinoma

Jaundice, itch

Antihistamines are not very effective Use soap-free body wash

Moisturize skin daily

Malnourishment
Adherence with lactulose (with or without rifaximin) Do not drink alcohol

Avoid sleeping tablets and opiate analgesics

Do not drive unless your doctor has told you it is okay to do so

Medication assistance may be required in severe memory disturbance

Highly educated and/or engaged participants:

discuss accumulation of ammonia and other toxins
Be mindful of worsening mood disturbances, personality changes, and sleep inversion

Family member or carer to help monitor for signs of deterioration

Titrate lactulose dose to achieve two or three loose bowel motions daily; reduce/withhold if diarrhea or severe bloating occurs

Violent, irrational behavior or loss of consciousness_call an ambulance immediately

Attend follow-up ultrasound appointments as scheduled

Get blood tests as directed

Acute or recurrent yellowing of eyes and skin - see a doctor as soon as possible

Weigh self once weekly

Keep a food diary

See a dietitian for expert advice
Adherence with propranolol (with or without proton pump inhibitor)

Highly educated and/or engaged participants: discuss portal hypertension and development of varices
Attend endoscopic surveillance as scheduled Monitor blood pressure, postural symptoms, dizziness Check hemoglobin and iron stores as appropriate Hematemesis or melena_call an ambulance immediately 
current medications and identify MRPs. The pharmacist will use open and closed questions to ascertain adherence, other medication-taking behaviors, and medication needs on an individualized basis. Participating patients will receive medication- and lifestyle-related education and advice tailored to their needs (decompensation history and medications) and receptiveness (Table 2). The pharmacist will also collaborate with the patient's general practitioner and hepatologist to optimize therapy, facilitate resolution of MRPs, and monitor issues where required.

Subsequent contact with participants will be made at 4-6 weeks $\left(t_{1}\right)$ and 12-14 weeks $\left(t_{2}\right)$ after the baseline interview to discuss changes in prescribed therapy; identify new MRPs and previously identified MRPs that are ongoing; reiterate advice provided at baseline; and provide additional relevant education about medications, lifestyle modifications, and self-management of cirrhosis to build on previous discussions. Promotion of a patientclinician partnership will be central to each discussion to encourage functional health literacy and self-efficacy with regard to patient confidence to raise questions and discuss issues with health care providers.

\section{Medication-related problems}

During patient interviews, the clinical pharmacist will identify actual and potential MRPs and document these in the patients' medical notes and on a structured data collection form. The pharmacist will then facilitate the resolution or monitoring of individual MRPs with other relevant clinicians as appropriate and document each outcome in patients' medical notes and data collection forms.

The clinical significance of MRPs (and medication discrepancies) will be assessed by a clinician panel using a risk matrix (Fig. 2) modified from the Metro North Health Service Risk Management Framework [19]. The matrix assigns a composite risk of prospective severity and likelihood of potential harm, as well as duration of time until potential harm may occur, as a consequence

Step 1:

Determine the RISK score using the SEVERITY AND LIKELIHOOD matrix

\begin{tabular}{|c|c|c|c|c|c|c|}
\hline \multirow[b]{2}{*}{$\begin{array}{l}\text { What is the } \\
\text { SEVERITY }\end{array}$} & & \multirow[b]{2}{*}{ Negligible } & \multirow[b]{2}{*}{ Minor } & \multirow[b]{2}{*}{ Moderate } & \multirow[b]{2}{*}{ Severe } & \multirow[b]{2}{*}{ Catastrophic } \\
\hline & & & & & & \\
\hline \multirow{2}{*}{$\begin{array}{c}\text { of the potential } \\
\text { harm? }\end{array}$} & Certain & Medium & Medium & High & Very High & Very High \\
\hline & Probable & Medium & Medium & High & High & Very High \\
\hline \multirow{2}{*}{$\begin{array}{l}\text { What is the } \\
\text { LIKELIHOOD }\end{array}$} & Possible & Low & Medium & High & High & High \\
\hline & Unlikely & Low & Medium & Medium & Medium & High \\
\hline of the potential & Rare & Low & Low & Low & Medium & High \\
\hline harm occurring? & & & & RISK & & \\
\hline
\end{tabular}

Step 2:

Determine overall SIGNIFICANCE using the RISK and TIMEFRAME matrix where;

A - Long Term Risk (i.e. months to years)

B - Intermediate Term Risk (i.e. weeks to months)

C - Short Term Risk (i.e. days to weeks)
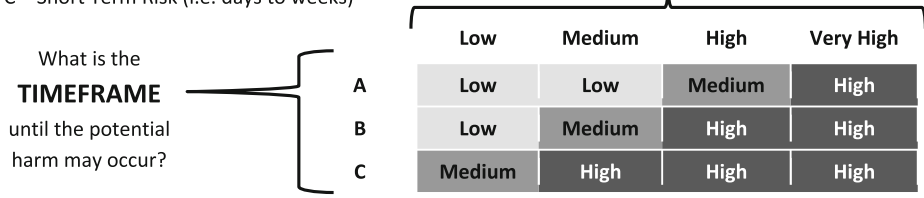

Step 3:

SIGNIFICANCE

Action based on SIGNIFICANCE assessment

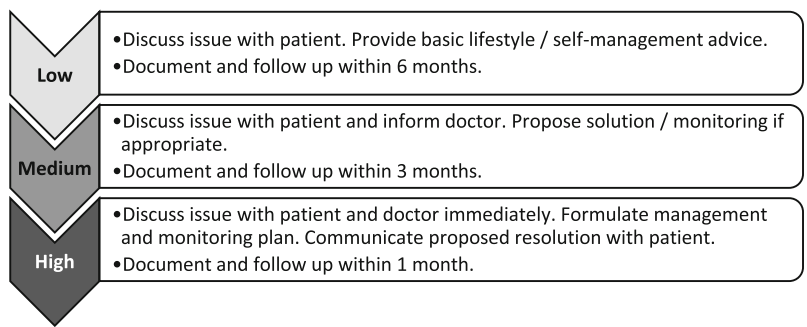

Fig. 2 Risk matrix for assessment of significance of medication discrepancies and other medication-related problems 
of the MRP. The panel of clinicians will comprise at least one clinical pharmacist, hepatologist, and general physician/pharmacologist who have experience in managing patients with cirrhosis. MRPs will be de-identified, randomized, and independently assessed by at least two members of the panel. Relevant clinical information will accompany the de-identified MRPs, but not details of patient outcomes or MRP resolution, to ensure unbiased prospective assessment of potential harm. Consensus of individual rankings will be used to determine the final measure of potential harm. Where there is disagreement between individual rankings, a roundtable panel discussion will be called to facilitate consensus.

\section{Tailored intensity intervention}

Intervention participants will receive education and advice tailored to their needs, receptiveness, and cognitive barriers. For example, a patient who has nonalcoholic steatohepatitis with well-controlled abdominal ascites and no other decompensation history would likely require a low-intensity intervention if he or she identified as adherent. Low-intensity intervention for this patient would consist of reiteration of important dietary modifications, monitoring of weight, and diuretic education. Conversely, a malnourished ex-alcoholic patient with a history of poorly controlled ascites, recurrent encephalopathy, and persistent hypotension will require highintensity intervention. This would ideally involve inclusion of a carer/family member in the discussion to educate both parties on the potential precipitants and early signs of encephalopathy, self-titration of lactulose, and when to present to the emergency department. Pharmacotherapy education (including possible discussion of adherence aids), high-protein and low-sodium dietary modifications, daily weight monitoring (including when to contact the clinic if weight is changing significantly over short periods of time), and review of pharmacotherapy that may contribute to hypotension would also be conducted. Liaison with additional health providers may also be required.

After providing education and advice to intervention participants, the clinical pharmacist will use a structured data collection form to record topics that have been discussed, topics that were not discussed and reasons why, and whether a carer was present. Post hoc testing will be employed to explore study outcomes according to the intensity of the intervention received and the completeness of relevant information provided (see the "Data analysis" section below).

\section{Medication reconciliation}

Using information from several sources (including the patient, general practitioner, pharmacy, own medications, carer), the clinical pharmacist will construct a reconciled list of current pharmacotherapy. The reconciled list of medications will be made available to other clinicians in the patient's medical record, and a patient-friendly guide will be constructed using the approved statewide template. This guide will be printed and provided to the patient. The patient will be encouraged to take it to all health care appointments and use it at home to aid with compliance and memory. The guide will also contain additional medication management and lifestyle points related to prescribed therapy, such as low-salt diet reminders for patients who are prescribed diuretics in the management of ascites. Patients will be encouraged to manually edit the medication guide when therapy is altered.

\section{Study endpoints and data collection}

Study outcomes, tools, and time points for measurement are outlined in Table 3. Follow-up will occur approximately 6 months post-enrollment (allowed range 140224 days after baseline interview), according to outpatient hepatology scheduling availability. If a follow-up appointment is missed, a second appointment will be offered to the patient within the allowed time frame. If the second appointment is missed, the patient will be considered lost to follow-up for $t_{3}$ survey endpoints.

\section{Primary outcome Medication discrepancies}

Discrepancies between patient-reported and cliniciandocumented current medications will be obtained via the patient questionnaire (medication recall), patient interview (reconciliation for intervention participants only), and medical record review (clinician documentation). The clinical significance of discrepancies will be determined by blinded comparison at the completion of the study using a risk matrix tool (Fig. 2), which assigns a composite ranking of severity, likelihood, and duration, as described in the Methods subsection "Medication-related problems" above. Discrepancies (between medications, doses, and frequency of administration) will be examined at baseline and follow-up $\left(t_{3}\right)$ for both groups. A composite endpoint of frequency (counts of) and potential risk will be examined by measuring total frequency of high/very high risk discrepancy occurrence and the frequency of high/very high risk discrepancy occurrence per patient.

\section{Secondary outcomes}

A questionnaire will be used to collect data on the secondary outcomes of interest at baseline and follow-up $\left(t_{3}\right)$. Validated tools used to measure these endpoints will be included in the questionnaires with approval from their respective developers. 
Table 3 Outcomes and other measures of the medication management and education intervention

\begin{tabular}{|c|c|c|}
\hline Categories/variables & Measure & Collection week \\
\hline \multicolumn{3}{|l|}{ Medication discrepancies } \\
\hline $\begin{array}{l}\text { Between drug name and dosage } \\
\text { Frequency of per-patient discrepancies } \\
\text { Severity of discrepancies }\end{array}$ & $\begin{array}{l}\text { Patient interview } \\
\text { Questionnaire } \\
\text { Medical record review }\end{array}$ & $t_{0}, t_{3}$ \\
\hline \multicolumn{3}{|l|}{ Medication-related problems (intervention arm only) } \\
\hline $\begin{array}{l}\text { Adverse drug reaction } \\
\text { Drug interaction } \\
\text { Untreated, undertreated, or overtreated indication } \\
\text { Other medication-related barriers }\end{array}$ & $\begin{array}{l}\text { Patient interview } \\
\text { Questionnaire }\end{array}$ & $t_{0}, t_{3}$ \\
\hline \multicolumn{3}{|l|}{ Adherence } \\
\hline $\begin{array}{l}\text { Self-reported medication adherence } \\
\text { Affordability }\end{array}$ & $\begin{array}{l}\text { MMAS-8 } \\
\text { Patient interview }\end{array}$ & $t_{0,}, t_{3}$ \\
\hline \multicolumn{3}{|l|}{ Quality of life } \\
\hline $\begin{array}{l}\text { Health-related quality of life } \\
\text { Abdominal and systemic symptoms } \\
\text { Emotional functioning, fatigue, and worry } \\
\text { Daily activity }\end{array}$ & $\begin{array}{l}\text { CLDQ } \\
\text { Brief IPQ }\end{array}$ & $t_{0,} t_{3}$ \\
\hline \multicolumn{3}{|l|}{ Beliefs and perceptions } \\
\hline $\begin{array}{l}\text { General medication beliefs } \\
\text { Beliefs about liver disease medications } \\
\text { Illness perceptions }\end{array}$ & $\begin{array}{l}\text { BMQ-General } \\
\text { BMQ-Specific } \\
\text { Brief IPQ }\end{array}$ & $t_{0,} t_{3}$ \\
\hline \multicolumn{3}{|l|}{ Knowledge/health literacy } \\
\hline $\begin{array}{l}\text { Knowledge of causation } \\
\text { Dietary modification including sodium restriction } \\
\text { Self-monitoring requirements } \\
\text { Safe analgesia } \\
\text { Source of medication-related information }\end{array}$ & $\begin{array}{l}\text { Questionnaire } \\
\text { Brief IPQ }\end{array}$ & $t_{0,} t_{3}$ \\
\hline \multicolumn{3}{|l|}{ Hospitalization } \\
\hline $\begin{array}{l}\text { Liver-related hospitalization } \\
\text { Non-liver-related hospitalization } \\
\text { Duration of stay }\end{array}$ & Medical record review & $\begin{array}{l}t_{3,} t_{4} \\
\text { (and } 3 \text { years) }\end{array}$ \\
\hline \multicolumn{3}{|l|}{ Survival } \\
\hline $\begin{array}{l}\text { Liver-related mortality } \\
\text { Non-liver-related mortality }\end{array}$ & Medical record review & $\begin{array}{l}t_{3,} t_{4} \\
\text { (and } 3 \text { years) }\end{array}$ \\
\hline \multicolumn{3}{|l|}{ Cost } \\
\hline $\begin{array}{l}\text { Cost of service implementation } \\
\text { Potential cost savings }\end{array}$ & Cost modeling & After study completion \\
\hline
\end{tabular}

Abbreviations: BMQ Beliefs about Medicines Questionnaire, Brief IPQ Brief Illness Perception Questionnaire, CLDQ Chronic Liver Disease Questionnaire, MMAS-8 Eight-question Morisky Medication Adherence Scale

\section{Adherence}

Change in self-reported medication adherence will be examined using the eight-question Morisky Medication Adherence Scale (MMAS-8) [20-22] and patient interview. The MMAS- 8 has been validated extensively in other chronic diseases and multiple languages worldwide [21-23]. It contains seven questions with yes/no alternatives and one question that features a 5-point Likert scale. Adherence scores can range from 0 to 8 and may be trichotomized into high (score 8), medium (score 6 to $<8$ ), and low (score $<6$ ) adherence rankings.

\section{Quality of life}

The Chronic Liver Disease Questionnaire (CLDQ) [24] is a widely used health-related QoL tool that has been validated in numerous etiologies and severities of CLD and in several languages [24-29]. The CLDQ asks patients to rank a series of symptoms, impacts on daily activities, and mood disturbances for frequency of occurrence using a 7-point Likert scale, where a higher score indicates a better QoL. It includes 29 items across 6 domains: 8 questions related to emotional function; 3 questions related to abdominal symptoms; 3 questions related to activity; and 5 
questions within each of the domains of systemic symptoms, fatigue, and worry.

\section{Medication beliefs}

Beliefs about medications will be examined using the Beliefs about Medicines Questionnaires (BMQ-General and BMQ-Specific) [30], which contain a series of statements about medications. Patients are required to indicate the extent of their personal agreement or disagreement with each statement using a 5-point Likert scale. The BMQ-General contains four items related to the overuse of medicines and four items about medication harmfulness. The BMQ-Specific contains five items about "necessity" beliefs and five items about "concerns" that patients may have about their medicines. The internal consistency, discriminant validity, and correlation of the BMQ with other clinical measures are described elsewhere [30-32].

\section{IIIness perceptions}

The Brief Illness Perception Questionnaire [33] will be used to examine changes in patients' perceptions about cirrhosis. The questionnaire has proven validity and correlation with outcomes in numerous chronic diseases $[33,34]$. It contains eight items that patients rank from 0 to 10 related to their perception of disease and medicines. Patients are also asked to identify three important factors that they believe caused their current illness.

\section{Cirrhosis and lifestyle knowledge}

Knowledge of cirrhosis self-management tasks related to decompensation history and prescribed medications will be determined via questionnaire (using the same questions pre- and postintervention). Eight questions related to over-the-counter analgesia, management of ascites and sodium restriction, and monitoring of weight and blood pressure have been incorporated into the surveys, adapted from Volk et al. [3].

\section{Hospitalization and mortality}

The number of hospital admissions, including the frequency of abdominal paracenteses in patients with ascites, will be derived from medical records. Hospitalizations for liver-related and non-liver-related events in the 2 years preceding recruitment and 12 months following recruitment, including data on patients' clinical decompensation prior to enrollment and postenrollment (e.g., date, type and severity of decompensation event, new or recurrent event) will be collected. Follow-up will be conducted at 12 months and again at 3 years. Frequency and duration of hospitalization for management of cirrhosis-related and non-cirrhosisrelated complications, cirrhosis-related mortality, and noncirrhosis-related mortality will be interpreted within the context of patients' liver disease severity, decompensation history, development of new decompensation events, and other prognostic and clinical variables.

\section{Medication-related problems}

Intervention participants may have MRPs identified during their interview that warrant follow-up action or monitoring. The pharmacist will document the types of MRPs identified, the action or monitoring required, and the steps taken toward resolution in patients' medical records and using the structured data collection tool. This information will not be available for usual care participants, because they will not have a medication review. The change in frequency (count) and potential harm (risk matrix) of MRPs will be assessed using an equivalent time sample where the intervention group is its own control. The clinical significance of MRPs will be determined by blinded comparison at the completion of the study using a risk matrix tool (Fig. 2), as described in the Methods subsection "Medication-related problems" above.

\section{Cost-effectiveness analyses}

Economic evaluation of the study intervention (with respect to potentially prevented hospital admissions, harms, reduced mortality, quality-adjusted life-years gained, and so forth) will be performed in collaboration with health economic analysts at the conclusion of the study.

\section{Safety measures for serious adverse events}

Study progress, collected data, and clinical cases will be reviewed periodically by the study investigators. Frequency and causation of participant hospitalization and mortality will be reviewed at 6,12 , and 18 months because the study group of interest is at high risk for these occurrences. If significant differences are found between the intervention and control groups, or if serious adverse events develop throughout the study, an independent data safety board will be convened to review the appropriateness of continuing the study in accordance with the Australian National Health and Medical Research Council National Statement on Ethical Conduct in Human Research [35].

\section{Intervention personnel Clinical pharmacist}

The intervention will be conducted by a tertiary hospitaltrained clinical pharmacist who has experience working in a hepatology unit and has received education from a hepatologist regarding symptoms, signs, and clinical significance of liver decompensation events. Ongoing continuous education relevant to hepatology and the management of liver patients will be undertaken throughout the study period and will consist of weekly meetings with hepatology 
consultants and advanced trainees as well as attendance at departmental case discussions, journal club meetings, and in-service education.

\section{Senior clinician supervision}

The intervention will be facilitated by specialist medical and nursing staff to provide clinical guidance and supervision to the intervention pharmacist. The pharmacist will work in collaboration with the senior hepatologists and the hepatology clinical nurse coordinator to identify and resolve MRPs and optimize therapy. At least one supervising hepatologist will be present in the clinic during the pharmacist's working hours. Recommendations to modify therapy will not be communicated to the patient without prior discussion with a supervising clinician. The study protocol and patient education provided to participants have been approved by the clinical leadership.

\section{Data analysis}

Data will be coded and stored in a password-protected database and analyzed using a statistical software package (IBM SPSS Statistics version 20.0; IBM, Armonk, NY, USA). Study endpoints experienced for each treatment group will be formally analyzed on an intentionto-treat basis [36]. Important potential confounders will be included in a secondary multivariate analysis (e.g., hospital admission, mortality). Post hoc subgroup analysis will be used to further explore outcomes according to the intensity of the intervention and completeness of relevant personalized education received by intervention participants.

The study endpoints will be presented quantitatively (proportions, statistical differences between groups) and qualitatively (description of discrepancies, MRPs, and some patient-reported medication beliefs and illness perceptions). All tests will be two-tailed, and significance will be set at $\alpha=0.05$. Continuous and normallydistributed variables will be presented as mean \pm SD. Differences between groups will be analyzed using oneway analysis of variance. Non-normally distributed data will be presented as median (range), and nonparametric tests will be used to analyze differences between groups. Categorical data will be analyzed using Pearson's chisquare test or Fisher's exact test. Pearson's correlation or Spearman's rank correlation coefficient will be used to identify linear relationships between normally distributed and non-normally distributed continuous variables, respectively.

Differences in the study endpoints of interest will be examined between the intervention and control groups at baseline and at follow-up. The endpoints will be examined at baseline to test for homogeneity between groups (effectiveness of randomization) and to identify clinical variables that may influence endpoints (e.g., Model for End-Stage Liver Disease score; Child-Turcotte-Pugh classification; decompensation history, including event date, type, severity, and recurrence; hospitalization frequency; age; sex; biochemical abnormalities; and other prognostic variables). Clinically significant differences at baseline between groups will be adjusted for during the final analysis. Changes in the frequency of medication discrepancies, self-reported adherence, QoL, medication beliefs, illness perceptions, and patient knowledge at follow-up will be examined to measure the effect of the intervention on study outcomes at group and individual levels. The primary endpoint of medication discrepancies will be explored using total frequency of instances and frequency of instances per patient and per medication group. Comparison between intervention and control groups at baseline and $t_{3}$ will be made using total number of instances per group, total number of high/very high risk instances per group, mean/median number of instances per patient, and mean/median number of high/very high risk instances per patient.

Predictors of clinical outcomes will be identified using binary logistic regression to determine the OR (95\% CI). A multivariable logistic regression model will be used to determine independent predictors for patient outcomes. The multivariable logistic regression model will be constructed using variables of clinical significance (severity of disease; decompensation history, including date, type, severity, and frequency of events; other prognostic variables) and statistical significance as determined by univariate analysis.

Time to first episode of decompensation and time to death (cause-specific and all-cause) will be assessed using Kaplan-Meier survival curves. The curves will be compared with the log-rank test statistic. Cox proportional hazards modeling will be used to calculate HRs, after testing the proportional hazards assumption. Patients will contribute person-time from the date of recruitment until the earliest of end of follow-up (12 months) or death (date of death to be obtained from medical records). Competing risks analysis will be performed in which we encode, as separate events, time to first readmission with a cirrhosis-related episode, death resulting from cirrhosis, or death resulting from other causes. This will be extended to multivariate analysis if there are a sufficient number of events.

It is anticipated that a proportion of participants (approximately $30 \%$ ) will be lost from the study before completing follow-up assessments at $t_{3}$. Although the sample size calculation has been adjusted to accommodate for this loss, subjects who have completed only one assessment will be included in the analyses to establish baseline parameters in both treatment groups. With respect to changes from baseline, data imputation will be 
considered. In decompensated cirrhosis, dropouts due to death are informatively censored, and thus the responses of patients with missing data will be modeled explicitly. Subjects will be matched using baseline demographic and patient-reported outcomes, repeating this technique for both intervention arms. Imputation methods such as a hot-deck imputation and iterative regression imputation will be used. The sensitivity of the results to each of these methods will be assessed to quantify the degree of bias present.

\section{Power calculation and sample size determination}

On the basis of a primary endpoint of a $50 \%$ reduction in medication discrepancies $(3.12 \pm 2.5$ per patient [6] to 1.56 per patient), 41 participants will be required per treatment arm to achieve $\geq 80 \%$ statistical power. Median survival in decompensated cirrhosis is 2 years [37]. Therefore, a 6-month mortality rate between $10 \%$ and $15 \%$ among the study cohort is predicted. The Hepatology Outpatient Centre's attendance failure/reschedule rate is approximately $20 \%$. Therefore, a possible $30 \%$ loss will be accounted for, and at least 106 participants will be recruited.

\section{Discussion}

Authors of meta-analyses and researchers in randomized controlled trials have found that clinical pharmacist interventions improved achievement of therapeutic targets (via improved adherence and medication titration) in people with diabetes [10], hypertension [38, 39], and hyperlipidemia [40]. In heart failure, pharmacist intervention identified and resolved MRPs [11], improved adherence [11], optimized medication titration [11, 41], and reduced all-cause mortality and heart failure-related hospitalizations [12]. Similar benefits were identified in renal and transplant groups [9, 13]. Addressing MRPs, discrepancies, nonadherence, and patient knowledge deficits related to prescribed therapy may also improve outcomes for patients with decompensated cirrhosis.

Volk et al. hypothesized that improved patients' understanding of their medications may prevent a significant proportion of 30-day readmissions among patients with decompensated cirrhosis [2]. However, patient understanding of disease and therapy is a difficult variable to ascertain and modify because it may be affected by numerous interacting dynamic domains, including health literacy, beliefs about health and medicines, patientclinician interaction (including quality of education and communication), self-efficacy, and the influence of other internal and external barriers [31, 32, 42]. These barriers, which may be social or economic factors, clinician or health care system factors, or patient-related factors, can further impact adherence [43]. People with poor health literacy, those living in disadvantaged areas, or those from culturally and linguistically diverse backgrounds comprise a large proportion of the CLD cohort [44-47]. These patients often have poor comprehension of chronic disease, including limited ability to access and use health information to make effective decisions about their health care without guidance [48]. Therefore, the intervention requires flexibility in its delivery and intensity to accommodate patients from a broad range of backgrounds.

Medication education, reconciliation of the medication history, and identification of MRPs are considered valuable clinical contributions by pharmacists to a patients' care [14]. Medication discrepancies have been identified in over $50 \%$ of ambulatory patients with cirrhosis and were associated with poorer adherence and polypharmacy [6]. MRPs have not been formally investigated in decompensated liver disease, but prevalence is thought to be high. Up to $12 \%$ of medical admissions annually in Australia have been associated with MRPs [15]; however, the risk of medication errors may be further compounded in people with advanced cirrhosis by impaired alertness and memory due to low-level chronic encephalopathy. This reduced cognitive activity can negatively impact disease insight, memory, and medication adherence. The education and medication management intervention must consider these potential cognitive barriers and involve the carer or a family member where appropriate.

Low health literacy has not been a barrier to improving health knowledge and medication management behaviors, though strategies and interventions to promote self-efficacy have been more effective in improving clinical status than information provision alone [42]. Illness and medication beliefs have been shown to influence patients' confidence to self-manage alcoholic liver disease and to have impacts on health-related QoL [49]. Progression of disease is associated with decreased QoL, including increased symptoms; abdominal symptoms and fatigue have been found to worsen adherence in people with cirrhosis [50]. Investigation of the effect of these variables on medication adherence in patients with decompensated cirrhosis, in addition to the effect of a clinical pharmacist education and medication management intervention on modifying these variables, will provide valuable information for future ambulatory management of these patients.

\section{Limitations}

In this study, we will evaluate the effectiveness of a single pharmacist in one general hepatology clinic in Australia, and applicability of findings to other sites will be dependent on similarities in patient demographics and models of care. An important component of this study is the provision of an intervention that is tailored 
to the patient's needs, receptiveness, and cognitive barriers. As a consequence of the "reactive" nature of the intervention, the information provided will be selective, and delivery will be staggered over the course of the study. Perceived variability in delivery may be due to lack of clinical indication (i.e., not providing information on precipitants of hepatic encephalopathy to patients who have never had encephalopathy), time restraints, patient disinterest, or "information overload." Where possible, advice and education points will be reiterated and built upon during future contacts.

There will be heavy reliance on patients' self-reported information, such as the details of their local pharmacy and general practitioner for the process of medication reconciliation; self-reported adherence; QoL; beliefs; and perceptions. This and other clinical information will also be dependent on honest patient responses to the survey questionnaires. Although this is acknowledged as a limitation, patient reporting remains the most important method of information gathering in clinical practice in terms of eliciting history and clinical status. Furthermore, self-reported adherence using the MMAS- 8 has been shown to be equally as accurate as and less onerous than other methods of adherence measurement, including pill counts. Where necessary, supplemental information will be sought from patients' family members or other carers to corroborate patients' responses if the study clinicians have concerns about patient safety in accordance with good clinical practice.

\section{Trial status}

At the time of proof review, recruitment into the study is complete.

\section{Additional file}

Additional file 1: SPIRIT 2013 checklist [18]: recommended items to address in a clinical trial protocol and related documents. Page numbers identified within the checklist correspond to the location of specified Standard Protocol Items within the original manuscript. (DOC $120 \mathrm{~kb}$ )

\section{Abbreviations}

BMQ: Beliefs about Medicines Questionnaire; Brief IPQ: Brief Illness Perception Questionnaire; CLD: Chronic liver disease; CLDQ: Chronic Liver Disease Questionnaire; MMAS-8: Eight-question Morisky Medication Adherence Scale; MRP: Medication-related problem; NSAID: Nonsteroidal anti-inflammatory drug; PAH: Princess Alexandra Hospital; QoL: Quality of life; SPIRIT: Standard Protocol Items: Recommendations for Interventional Trials

\section{Acknowledgements}

The authors acknowledge the consultant hepatologists and fellows, clinical nursing staff, and the clinical leadership within the Department of Gastroenterology and Hepatology at the Princess Alexandra Hospital for their support in the development and implementation of this project, in particular, Dr Katherine Stuart, Dr Caroline Tallis, Dr Paul Clark, Dr Lara Kane, A/Prof Graeme Macdonald, Dr Rebecca Ryan, Prof Gerald Holtmann, Penny Wright, Marrianne Black and Kate Choi. The authors also recognize the valuable technical supported provided by Valerie Logan.
Funding

PCV was supported by the Australian National Health and Medical Research Council (Career Development Fellowship 1083090).

\section{Availability of data and materials}

Scientists wishing to access materials used in the development of the intervention for noncommercial purposes, including the survey tools used, may contact the corresponding author directly.

\section{Authors' contributions}

KLH conceived of and designed the study, obtained ethical clearance, registered the trial, drafted the manuscript and assisted with study implementation. JHM and WNC assisted with study design, particularly planning the clinical impacts and analyses, and study implementation. AK was responsible for data entry and maintenance. LUH assisted with ethics applications and study implementation. PJP provided essential clinical support and supervision. DDS and KMI assisted with the statistical design of the study and provided other technical support. EEP and PCV assisted with study design, particularly clinical impacts and analyses, and study implementation, including essential clinical and technical support. PCV assisted in writing the manuscript. The manuscript was reviewed and approved by all authors.

\section{Ethics approval and consent to participate}

Ethics approval was received for the study protocol from the Metro South Hospital and Health Service Human Research Ethics Committee (HREC/15/ QPAH/688) and the University of Queensland Medical Research Ethics Committee (UQ2016000032) prior to commencement. Informed written consent will be obtained from each participant (and their formal carer/ power of attorney as appropriate). A copy of the signed consent form will be filed in each patient's medical record. The trial was retrospectively registered with the Australian and New Zealand Clinical Trial Registry on 15 June 2016 (ACTRN12616000780459).

\section{Consent for publication}

Not applicable, because individuals' data are not identifiable.

\section{Competing interests}

The authors declare that they have no competing interests.

\section{Publisher's Note}

Springer Nature remains neutral with regard to jurisdictional claims in published maps and institutional affiliations.

\section{Author details}

'School of Medicine, The University of Queensland, Brisbane, QLD, Australia. ${ }^{2}$ Pharmacy Department, Princess Alexandra Hospital, Brisbane, QLD, Australia. ${ }^{3}$ School of Medicine and Public Health, The University of Newcastle, Newcastle, NSW, Australia. ${ }^{4}$ School of Pharmacy, The University of Queensland, Brisbane, QLD, Australia. ${ }^{5}$ The Centre for Liver Disease Research, Translational Research Institute, The University of Queensland, Brisbane, QLD, Australia. ${ }^{6}$ Department of Gastroenterology and Hepatology, Princess Alexandra Hospital, Brisbane, QLD, Australia. ${ }^{7}$ Statistics Unit, QIMR Berghofer Medical Research Institute, Brisbane, QLD, Australia. ${ }^{8}$ Cancer and Chronic Disease Research Group, Level 4, Central, QIMR Berghofer Medical Research Institute, 300 Herston Rd, Brisbane, QLD 4006, Australia.

Received: 16 September 2016 Accepted: 1 July 2017

Published online: 20 July 2017

\section{References}

1. Ganesh S, Rogal SS, Yadav D, Humar A, Behari J. Risk factors for frequent readmissions and barriers to transplantation in patients with cirrhosis. PLoS One. 2013;8, e55140.

2. Volk ML, Tocco RS, Bazick J, Rakoski MO, Lok AS. Hospital readmissions among patients with decompensated cirrhosis. Am J Gastroenterol. 2012; 107:247-52

3. Volk ML, Fisher N, Fontana RJ. Patient knowledge about disease selfmanagement in cirrhosis. Am J Gastroenterol. 2013;108:302-5.

4. Hayward KH, Horsfall LU, Ruffin B, Cottrell WN, Chachay VS, Irvine KM, et al. Optimising care of patients with chronic disease: patient-oriented education 
may improve disease knowledge and self-management. Intern Med J. 47:8 doi:10.1111/imj.13505.

5. Fagan KJ, Zhao EY, Horsfall LU, Ruffin BJ, Kruger MS, McPhail SM, et al. Burden of decompensated cirrhosis and ascites on hospital services in a tertiary care facility: time for change? Intern Med J. 2014;44:865-72.

6. Hayward KL, Valery PC, Cottrell WN, Irvine KM, Horsfall LU, Tallis CJ, et al. Prevalence of medication discrepancies in patients with cirrhosis: a pilot study. BMC Gastroenterol. 2016;16:114.

7. Ha JF, Longnecker N. Doctor-patient communication: a review. Ochsner J. 2010;10:38-43.

8. Hepler CD, Strand LM. Opportunities and responsibilities in pharmaceutical care. Am J Hosp Pharm. 1990:47:533-43.

9. Wang HY, Chan AL, Chen MT, Liao CH, Tian YF. Effects of pharmaceutical care intervention by clinical pharmacists in renal transplant clinics. Transplant Proc. 2008;40:2319-23.

10. Machado M, Bajcar J, Guzzo GC, Einarson TR. Sensitivity of patient outcomes to pharmacist interventions. Part I: systematic review and meta-analysis in diabetes management. Ann Pharmacother. 2007;41:1569-82.

11. Gastelurrutia P, Benrimoj SI, Espejo J, Tuneu L, Mangues MA, Bayes-Genis A. Negative clinical outcomes associated with drug-related problems in heart failure (HF) outpatients: impact of a pharmacist in a multidisciplinary HF clinic. J Card Fail. 2011;17:217-23.

12. Koshman SL, Charrois TL, Simpson SH, McAlister FA, Tsuyuki RT. Pharmacist care of patients with heart failure: a systematic review of randomized trials. Arch Intern Med. 2008;168:687-94.

13. Chisholm MA, Mulloy LL, Jagadeesan M, DiPiro JT. Impact of clinical pharmacy services on renal transplant patients' compliance with immunosuppressive medications. Clin Transplant. 2001;15:330-6.

14. Lo L, Kwan J, Fernandes OA, Shojania KG. Medication reconciliation supported by clinical pharmacists. In: Shekelle PG, Wachter RM, Pronovost PJ, Schoelles K, McDonald KM, et al., editors. Making health care safer II: an updated critical analysis of the evidence for patient safety practices. Comparative Effectiveness Review No. 211. AHRQ Publication No. 13-E001-EF. Rockville, MD: Agency for Healthcare Research and Quality; 2013. p. 270-86

15. Roughead L, Semple S, Rosenfeld E. Literature review: medication safety in Australia. Sydney: Australian Commission on Safety and Quality in Health Care; 2013.

16. Rockey DC, Caldwell SH, Goodman ZD, Nelson RC, Smith AD, American Association for the Study of Liver Diseases. Liver biopsy. Hepatology. 2009; 49:1017-44.

17. European Association for Study of Liver, Asociacion Latinoamericana para el Estudio del Higado. EASL-ALEH Clinical Practice Guidelines: Non-invasive tests for evaluation of liver disease severity and prognosis. J Hepatol. 2015;63:237-64.

18. Chan AW, Tetzlaff JM, Altman DG, Laupacis A, Gotzsche PC, Krleza-Jeric K, et al. SPIRIT 2013 statement: defining standard protocol items for clinical trials. Ann Intern Med. 2013;158:200-7.

19. Metro North Hospital and Health Service. Risk Management Framework Guide for Implementation. Version no. 2.0. Effective date: 08/2015:29-31.

20. Morisky DE, DiMatteo MR. Improving the measurement of self-reported medication nonadherence: response to authors. J Clin Epidemiol. 2011;64:255-7.

21. Morisky DE, Ang A, Krousel-Wood M, Ward HJ. Predictive validity of a medication adherence measure in an outpatient setting. J Clin Hypertens (Greenwich). 2008;10:348-54.

22. Krousel-Wood M, Islam T, Webber LS, Re RN, Morisky DE, Muntner P. New medication adherence scale versus pharmacy fill rates in seniors with hypertension. Am J Manag Care. 2009;15:59-66.

23. Tan X, Patel I, Chang J. Review of the four item Morisky Medication Adherence Scale (MMAS-4) and eight item Morisky Medication Adherence Scale (MMAS-8). Inov Pharm. 2014;5:Article 165.

24. Younossi ZM, Guyatt G, Kiwi M, Boparai N, King D. Development of a disease specific questionnaire to measure health related quality of life in patients with chronic liver disease. Gut. 1999;45:295-300.

25. Younossi ZM, Boparai N, Price LL, Kiwi ML, McCormick M, Guyatt G. Healthrelated quality of life in chronic liver disease: the impact of type and severity of disease. Am J Gastroenterol. 2001;96:2199-205.

26. Sobhonslidsuk A, Silpakit C, Kongsakon R, Satitpornkul P, Sripetch C. Chronic liver disease questionnaire: translation and validation in Thais. World J Gastroenterol. 2004;10:1954-7.

27. Ferrer M, Córdoba J, Garin O, Olivé G, Flavià M, Vargas V, et al. Validity of the Spanish version of the Chronic Liver Disease Questionnaire (CLDQ) as a standard outcome for quality of life assessment. Liver Transpl. 2006;12:95-104.
28. Zuberi BF, Memon AR, Afsar S, Qadeer R, Kumar R. Correlation of quality of life in patients of cirrhosis of liver with etiology and disease severity using disease-specific quality of life questionnaire. J Ayub Med Coll Abbottabad. 2007:19:7-11.

29. Chawla KS, Talwalkar JA, Keach JC, Malinchoc M, Lindor KD, Jorgensen R. Reliability and validity of the Chronic Liver Disease Questionnaire (CLDQ) in adults with non-alcoholic steatohepatitis (NASH). BMJ Open Gastroenterol. 2016:3, e000069.

30. Horne R, Weinman J, Hankins M. The Beliefs about Medicines Questionnaire: the development and evaluation of a new method for assessing the cognitive representation of medication. Psychol Health. 1999;14:1-24.

31. Horne R, Chapman SC, Parham R, Freemantle N, Forbes A, Cooper V. Understanding patients' adherence-related beliefs about medicines prescribed for long-term conditions: a meta-analytic review of the Necessity-Concerns Framework. PLoS One. 2013;8, e80633.

32. Alhewiti A. Adherence to long-term therapies and beliefs about medications. Int J Family Med. 2014;2014:479596.

33. Broadbent E, Petrie KJ, Main J, Weinman J. The brief illness perception questionnaire. J Psychosom Res. 2006;60:631-7.

34. Broadbent E, Wilkes C, Koschwanez H, Weinman J, Norton S, Petrie KJ. A systematic review and meta-analysis of the Brief Illness Perception Questionnaire. Psychol Health. 2015;30:1361-85.

35. National Health and Medical Research Council (NHMRC). National Statement on Ethical Conduct in Human Research 2007 [updated May 2015]. Canberra: NHMRC. https://www.nhmrc.gov.au/book/national-statement-ethicalconduct-human-research. Accessed 22 Jun 2016.

36. Meinert C, Tonascia S. Clinical trials: design, conduct and analysis. New York: Oxford University Press; 1986.

37. D'Amico G, Garcia-Tsao G, Pagliaro L. Natural history and prognostic indicators of survival in cirrhosis: a systematic review of 118 studies. J Hepatol. 2006;44:217-31.

38. Machado M, Bajcar J, Guzzo GC, Einarson TR. Sensitivity of patient outcomes to pharmacist interventions. Part II: Systematic review and meta-analysis in hypertension management. Ann Pharmacother. 2007;41:1770-81.

39. Simpson SH, Majumdar SR, Tsuyuki RT, Lewanczuk RZ, Spooner R, Johnson JA. Effect of adding pharmacists to primary care teams on blood pressure control in patients with type 2 diabetes: a randomized controlled trial. Diabetes Care. 2011;34:20-6.

40. Machado M, Nassor N, Bajcar JM, Guzzo GC, Einarson TR. Sensitivity of patient outcomes to pharmacist interventions. Part III: systematic review and meta-analysis in hyperlipidemia management. Ann Pharmacother. 2008;42: 1195-207.

41. Martinez AS, Saef J, Paszczuk A, Bhatt-Chugani H. Implementation of a pharmacist-managed heart failure medication titration clinic. Am J Health Syst Pharm. 2013;70:1070-6.

42. Osborn CY, Paasche-Orlow MK, Bailey SC, Wolf MS. The mechanisms linking health literacy to behavior and health status. Am J Health Behav. 2011;35:118-28.

43. Sabaté E. Adherence to long-term therapies: evidence for action. Geneva, Switzerland: World Health Organisation; 2003.

44. Hepatitis Australia. Hepatitis B testing and treatment in relevant culturally and linguistically diverse communities. Woden, Australia: Hepatitis Australia; 2014 http://www.hepatitisaustralia.com/position-papers/. Accessed 13 Jun 2016

45. Dahl TF, Cowie BC, Biggs BA, Leder K, MacLachlan JH, Marshall C. Health literacy in patients with chronic hepatitis B attending a tertiary hospital in Melbourne: a questionnaire based survey. BMC Infect Dis. 2014;14:537.

46. El-Atem N, Wojcik K, Horsfall L, Irvine K, Johnson T, McPhail SM, et al. Patterns of service utilisation within hepatology clinics: high prevalence of advanced liver disease. J Gastroenterol Hepatol. 2015;30:100-1.

47. Preston-Thomas A, Fagan $\mathrm{P}$, Nakata $Y$, Anderson E. Chronic hepatitis B: care delivery and patient knowledge in the Torres Strait region of Australia. Aust Fam Physician. 2013:42:225-31.

48. Australian Commission on Safety and Quality in Health Care (ACSQHC). Health literacy: taking action to improve safety and quality. Sydney: ACSQHC; 2014.

49. Lau-Walker M, Presky J, Webzell I, Murrells T, Heaton N. Patients with alcohol-related liver disease - beliefs about their illness and factors that influence their self-management. J Adv Nurs. 2016;72:173-85.

50. Polis S, Zang L, Mainali B, Pons R, Pavendranathan G, Zekry A, et al. Factors associated with medication adherence in patients living with cirrhosis. J Clin Nurs. 2016:25:204-12 